\title{
EVERY DIRECTION A JULIA DIRECTION
}

\author{
BRYAN E. CAIN
}

ABSTRACT. Let $f(z)=\exp (\cosh z)$. If $N$ is any $\epsilon$-neighborhood of any ray through the origin with slope $m \neq 0, \infty$ then $f^{-1}(w) \cap N$ is infinite if $w \neq 0$.

Let $J[f]$ denote the set of Julia directions of the entire function $f$. That is, $\theta \in J[f]$ if, in every sector $\alpha<\arg z<\beta$ such that $\alpha<\theta<\beta$, $f$ assumes every complex value, with at most one exception, infinitely often. Using infinite products Julia [2] has constructed an entire function for which every direction is a Julia direction. The example which follows is more elementary.

The closed annulus $\{1 / n \leq|z| \leq n\}$ will be denoted $A_{n}$ for $n=1,2$, $\cdots$. If $a, b, \delta>0$ the closed rectangle $\{x+i y:-a \leq x \leq a: b-\delta \leq y \leq b$ $+\delta\}$ will be denoted by $R_{\delta}(a, b)$.

Theorem. If $f(z)=\exp (\cosh z)$ then $J[f]=\mathbf{R}$.

Proof. The relations $f(\bar{z})=(f(z))^{-}$and $f(z)=f(-z)$ imply that if $\theta \in J[f]$ then $-\theta, \theta \pm \pi \in J[f]$. Consequently we can finish the proof by showing that $(0, \pi / 2) \subset J[f]$ because the set of Julia directions is clearly always closed.

Now suppose that $\theta, \alpha, \beta$ are given and that they satisfy $0<\theta<\pi / 2$ and $\alpha<\theta<\beta$. They will be fixed for the rest of the proof.

Let $S=\{x+i y: m x-\epsilon<y<m x+\epsilon\}$ where $m=\tan \theta$ and $0<\epsilon<\pi$. The function $z \rightarrow 1 / 2 \exp (z)$ maps the strip $S$ onto a ribbon which starts at the origin, wraps around it infinitely often, and spirals out to infinity. This ribbon has $\gamma_{-}$for its inside boundary and $\gamma_{+}$for its outside boundary where

$$
\gamma_{ \pm}(y)=1 / 2 \exp [(y \pm \epsilon) / m+i y]
$$

for all $y$. The width of the ribbon $\left|\gamma_{+}(y)-\gamma_{-}(y)\right|$, which is measured along a ray from the origin, is an unbounded increasing function of $y$. The ribbon does not overlap itself because $\left|\gamma_{-}(y+2 \pi)\right|-\left|\gamma_{+}(y)\right|>0$ provided $\epsilon<\pi$.

Received by the editors August 30, 1973.

AMS (MOS) subject classifications (1970). Primary 30 A70. 
Let $P_{n}$ denote the open parallelogram which is the intersection of the strip $\{x+i y: 2 \pi n<y<2 \pi n+\pi\}$ with the strip $S$. Note that for all $n$ larger than some $n_{0}$ the sector $\alpha<\arg z<\beta$ will contain $P_{n}$, and that the $P_{n}^{\text {'s }}$ are disjoint. Thus the proof can be finished by showing that if $w \neq 0$ then $w \in f\left(P_{n}\right)$ for infinitely many $n$.

Let ic ${ }_{n}$ be the midpoint of the interval in which $1 / 2 \exp \left(P_{n}\right)$ meets the imaginary axis. Since $1 / 2 \exp \left(P_{n}\right)$ is the interior of one component of the portion of the ribbon lying in the half plane $\operatorname{Im} z>0$, we know that $c_{n}>0$.

Lemma. If $a>0$ then $R_{\pi}\left(a, c_{k}\right) \subseteq \cosh \left(P_{k}\right)$ for infinitely many values of $k$.

Using this lemma we let $U_{n}=P_{k}$, where $k>n_{0}$ is chosen so large that $R_{\pi}\left(\log n, c_{k}\right) \subseteq \cosh \left(P_{k}\right)$ and $P_{k}$ is disjoint from $U_{1}, \cdots, U_{n-1}$. Then $f\left(U_{n}\right) \supseteq \exp \left(R_{\pi}\left(\log n, c_{k}\right)\right)=A_{n}$, and, since every $w \neq 0$ lies in infinitely many $A_{n}, f^{-1}(w) \cap\{\alpha<\arg z<\beta\}$ is infinite. Thus $\theta \in J[f]$.

Remark. This proof actually shows that if $\theta \neq 0$ or $\pi / 2(\bmod \pi)$ and if $N$ is any $\epsilon-n$ eighborhood of a ray from the origin through $e^{i \theta}$, then $f^{-1}(w) \cap$ $N$ is infinite if $w \neq 0$. Had we merely wished to prove the Theorem we could have replaced the $P_{n}$ 's with a sequence of disjoint rectangles $R_{n}=$ $\left\{x+i y: a_{n}<x<b_{n}, 2 \pi n<y<2 \pi n+\pi\right\}$ which lie in $\alpha<\arg z<\beta$ and for which the sequences $a_{n}$ and $b_{n}-a_{n}$ approach $\infty$. Then $1 / 2 \cdot \exp \left(R_{n}\right)$ is the intersection of the half plane $\operatorname{Im} z>0$ with the annulus $\exp \left(a_{n}\right)<|z|<$ $\exp \left(b_{n}\right)$. Since the width $\exp \left(a_{n}\right)-\exp \left(b_{n}\right)$ of the annulus approaches $\infty$, it is geometrically clear that if $a>0$ and if $c_{n}=1 / 2 \cdot\left(\exp \left(a_{n}\right)+\exp \left(b_{n}\right)\right)$, there will exist infinitely many $n$ 's for which the rectangle $R_{\pi}\left(a, c_{n}\right)$ lies inside $1 / 2 \exp \left(R_{n}\right)$. But when $a_{n}$ is large the boundary of $\cosh \left(R_{n}\right)^{n}$ will stay very close to the boundary of $1 / 2 \exp \left(R_{n}\right)$ because then $1 / 2 \cdot \exp \left(-R_{n}\right)$ must lie within a tiny neighborhood of 0 . Thus $R_{\pi}\left(a, c_{n}\right) \subseteq \cosh \left(R_{n}\right)$ for infinitely many $n$ 's. Using this version of the Lemma, the Theorem can be proved by replacing the parallelograms $P_{n}$ in the proof above with the rectangles $R_{n}$.

Proof of the Lemma. Suppose that $\delta>0$ and let $b_{n}$ be the largest number such that the interior of $R_{\delta}\left(b_{n}, c_{n}\right)$ is contained in $1 / 2 \exp \left(P_{n}\right)$. (Once the width of the ribbon exceeds $2 \delta, b_{n}$ will be positive.) Then some vertex of $R_{\delta}\left(b_{n}, c_{n}\right)$ lies on $\gamma_{+}$or $\gamma_{-}$, and we shall show that this implies the unboundedness of $\left\{b_{n}\right\}_{\text {. Since }}\left|\gamma_{ \pm}(y)\right|$ are increasing functions of $y$ there are just two cases: (1) the northeast vertex $b_{n}+i\left(c_{n}+\delta\right)$ lies on $\gamma_{+}$, or (2) the southwest vertex $-b_{n}+i\left(c_{n}-\delta\right)$ lies on $\gamma_{-}$.

When case (.1) holds we have 


$$
b_{n}+i\left(c_{n}+\delta\right)=\gamma_{+}\left(2 \pi n+\phi_{n}\right) \quad \text { where } \phi_{n}=\tan ^{-1}\left[\left(c_{n}+\delta\right) / b_{n}\right] .
$$

Assuming that $b_{n}$ is bounded implies that $\phi_{n} \rightarrow \pi / 2$ because $c_{n} \rightarrow \infty$. Now we divide equation $(A)$ by $\gamma_{+}(2 \pi n+\pi / 2)$. Since

$$
c_{n}=\frac{1}{2 i}\left(\gamma_{+}\left(2 \pi n+\frac{\pi}{2}\right)+\gamma_{-}\left(2 \pi n+\frac{\pi}{2}\right)\right) \text { and since } \frac{\gamma_{-}(y)}{\gamma_{+}(y)}=\exp \left[\frac{-2 \epsilon}{m}\right]
$$

the left side becomes

$$
\left[\frac{b_{n}+i \delta}{\gamma_{+}(2 \pi n+\pi / 2)}\right]+\frac{1}{2}\left(1+\exp \left[\frac{-2 \epsilon}{m}\right]\right) .
$$

The right side becomes $\exp \left[(1 / m+i)\left(\phi_{n}-\pi / 2\right)\right]$ and if case (1) obtains for infinitely many $n$ we can equate the limit as $n \rightarrow \infty$ of each side and produce the contradiction $1 / 2(1+\exp [-2 \epsilon / m])=1$.

Case (2) gives

(B) $-b_{n}+i\left(c_{n}-\delta\right)=\gamma_{-}\left(2 \pi n+\pi-\psi_{n}\right)$ where $\psi_{n}=\tan ^{-1}\left[\left(c_{n}-\delta\right) / b_{n}\right]$.

If $b_{n}$ is bounded and (B) holds infinitely often, then dividing by $\gamma_{-}(2 \pi n+\pi / 2)$ and letting $n$ approach $\infty$ makes $\psi_{n} \rightarrow \pi / 2$ and gives the contradiction $1 / 2 .(\exp (2 \epsilon / m)+1)=1$.

This proves that $b_{n}$ is unbounded. Thus, in particular, if $\delta=\pi+1$ and $b=a+1$ the inclusion $R_{\pi}\left(a, c_{n}\right) \subset R_{\delta}\left(b, c_{n}\right) \subset 1 / 2 \cdot \exp \left(P_{n}\right)$ holds for infinitely many values of $n$. Then when $n$ is large enough the set $1 / 2 \exp \left(-P_{n}\right)$ will lie in a neighborhood of 0 so small that $\cosh \left(P_{n}\right)$ very nearly contains $R_{\delta}\left(b, c_{n}\right)$ and certainly contains $R_{\pi}\left(a, c_{n}\right)$.

Acknowledgement. We are indebted to $\mathrm{Peter} \mathrm{Colwell}$ for provocative discussions and to Richard Tondra for improving our example.

\section{REFERENCES}

1. E. Hille, Analytic function theory. Vol. II, Introductions to Higher Math., Ginn; New York, 1962. MR 34 \# 1490.

2. G. Julia, Sur quelques propriétés nouvelles des fonctions entières ou méromorphes (liere Mémoire), Ann. Sci. École Norm. Sup. 36 (1919), 93-125.

3. T. Zinno, Some properties of Julia's exceptional functions and an example of Julia's exceptional functions with Julia's direction, Ann. Acad. Sci. Fenn. Ser. A I, No. 464 (1970), 12 pp. MR 43 \#6414.

DEPARTMENT OF MATHEMATICS, IOWA STATE UNIVERSITY, AMES, IOWA 50010

Current address: Mathematiches Institut der Technishen Universität München, 8 München 2, Arcisstrasse 21, Postfach 202 420, Federal Republic of Germany 